\title{
Per què l'aigua calenta de vegades es congela més ràpidament que l’aigua freda?
}

Raquel Rios Font (Raquel.Rios@uab.cat) Centre de Recerca per a l'Educació Científica i Matemàtica (CRECIM). Universitat Autònoma de Barcelona.

El juliol del 2012 la Royal Society of Chemistry va convocar un concurs en el qual s'oferia un premi de 1000 lliures a la persona o equip que donés la millor explicació a un fenomen que consisteix en el fet que l'aigua calenta, en determinades condicions, es congela més ràpidament que l'aigua freda. D'entre les 22000 propostes presentades, va guanyar l'article del croata Nikola Bregovic, on es discuteixen algunes de les possibles causes d'aquest fenomen, que encara no està del tot resolt.

Paraules clau: Mpemba, aigua, congelació, convecció, súper refredament.

\section{L’Efecte Mpemba: una breu visió his- tòrica}

El fet que l'aigua calenta de vegades trigui menys temps a congelar-se que l'aigua freda es coneix des de l'antiguitat, i ja havia estat observat per Aristòtil el segle IV a.C. Sembla ser que altres personatges com Francis Bacon o René Descartes van parar atenció a aquest fenomen, i durant molt de temps diversos científics han intentat explicar-lo sense que s'arribés a cap consens.

Fa uns cinquanta anys aquesta qüestió va tornar a prendre protagonisme quan un noi tanzà que es deia Erasto Mpemba va trobar que la barreja calenta de llet i sucre per fer gelats es congelava abans que la barreja freda. Després de la incredulitat inicial dels seus companys de classe i del seu professor de física, va aprofitar una visita que el professor Dr. Denis Osborne feia a l'institut on estudiava, per preguntar-li el següent: per què, si es posen al congelador dos recipients similars d'igual volum, un d'ells ple d'aigua a $35^{\circ} \mathrm{C}$ i l'altre ple d'aigua a $100^{\circ} \mathrm{C}$, el primer en congelar-se és el que inicialment estava a $100^{\circ} \mathrm{C}$ ? En un primer moment, el Dr. Osborne no va saber donar-li una resposta, i fins i tot va pensar que en Mpemba estava equivocat. Tot i així, creia que era necessari animar els estudiants a qüestionar i a desenvolupar actituds crítiques, i en aquest cas s'havia trobat amb un alumne que afirmava les seves observacions amb molta seguretat. Així doncs, va decidir investigar-ho ell mateix, i va realitzar diversos experiments que el van portar a corroborar allò que el jove Mpemba li havia dit, és a dir, que l'aigua calenta, de vegades, es congela més ràpidament que l'aigua freda. Els resultats van ser publicats per tots dos conjuntament a la revista Physics Education l'any 1969 [1], i en honor al jove tanzà, aquest fenomen rep el nom d'efecte Mpemba.

\section{El concurs sobre l’Efecte Mpemba}

Malgrat tractar-se d'un fenomen que es coneix des de fa molt de temps, el fet que l'aigua calenta pugui congelar-se abans que l'aigua freda és encara una qüestió no resolta i molt fascinant. Per aquest motiu, la Royal Society of Chemistry va considerar que oferia una bona oportunitat per despertar l'entusiasme per la química i obrir un debat sobre la comunicació i l'ensenyament d'aquesta disciplina d'una manera accessible, efectiva i inspiradora.

Així doncs, en el marc d'una escola d'estiu que va tenir Iloc a Londres el juliol del 2012, la Royal Society of Chemistry va convocar un concurs en què s'oferia un premi de 1000 lliures a qui donés la millor explicació a l'efecte Mpemba ${ }^{1}$. La participació estava oberta al públic en general i es podia fer servir qualsevol mitjà, incloent articles, vídeos o il-lustracions. L'èxit de la convocatòria va ser molt

\footnotetext{
${ }^{1}$ http://www.rsc.org/mpemba-competition/
} 
alt, tenint en compte que es van presentar al voltant de 22000 propostes de 122 països diferents ${ }^{2}$. Després de mesos de votacions públiques i de revisió per experts (peer review), es van seleccionar onze finalistes, entre els quals hi ha estudiants universitaris de química, de biotecnologia, d'enginyeria, etc., així com també gent sense cap formació específica en química. ${ }^{3}$ El dia 10 de gener va tenir lloc la cerimònia d'entrega de premis, on es va anunciar que el guanyador del concurs era el croata Nikola Bregovic, un químic que treballa al Departament de Química de la Universitat de Zagreb.

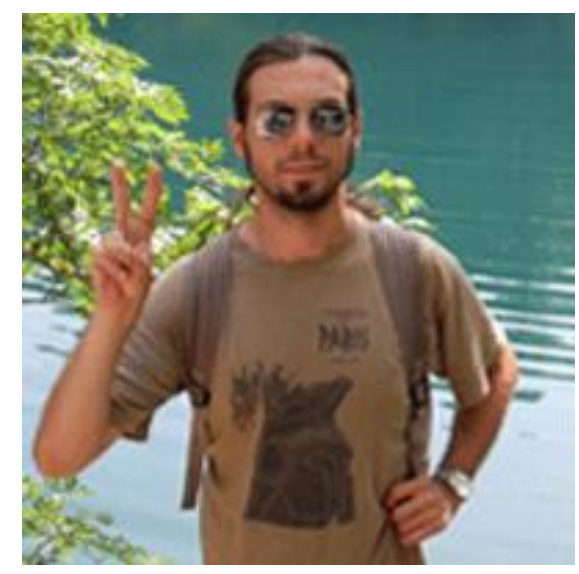

Figura 1. El croata Nikola Bregovic, guanyador del concurs sobre l'Efecte Mpemba convocat per la Royal Society of Chemistry el juliol del 2012.

\section{La proposta de Nikola Bregovic}

Tal i com explica en l'article de 9 pàgines que va presentar al concurs [2], va ser gràcies al correu d'un amic que va tenir coneixement sobre l'efecte Mpemba, la naturalesa del qual el va deixar molt intrigat. Aprofitant el fet que treballava en un laboratori a la universitat, va poder dur a terme una sèrie d'experiments (a la Figura 2 es mostra el resultat d'un d'ells), i al mateix temps va començar a llegir diversos informes $\mathrm{i}$ articles que s'havien publicat sobre el fet que l'aigua calenta es congeli més ràpidament que l'aigua freda ${ }^{4}$. A partir de la recerca

${ }^{2}$ Les xifres per a cada país es poden consultar a la web: http://prospect.rsc.org/mpemba-competition-map/

${ }^{3}$ Les seves propostes estan disponibles a la web: http://www.rsc.org/mpemba-competition/shortlist/

${ }^{4}$ També va trobar una pàgina web on es venen kits per realitzar l'experiment de l'efecte Mpemba: bibliogràfica, va trobar que aquest efecte era atribuït fonamentalment a quatre causes:

- L'evaporació de l'aigua

- Els gasos dissolts

- La convecció induïda per gradients de temperatura

- El súper refredament o subfusió (supercooling, en anglès)

En el seu article, Bregovic no aporta cap explicació nova respecte aquestes que han estat suggerides. El que sí fa és discutir en detall aquests quatre punts i, basant-se en un seguit d'experiments i en els seus coneixements de termodinàmica, dóna arguments per recolzar o refutar el fet que puguin ser o no causants de l'efecte Mpemba. Aquest efecte es pot observar a partir de les corbes de la Figura 2 , on veiem que la mostra que inicialment estava a més temperatura s'estabilitza a $0^{\circ} \mathrm{C}$ uns 10 minuts abans que la mostra que inicialment estava més freda. Un dels punts que remarca l'autor i que és interessant esmentar és que els resultats dels seus experiments posen de manifest un problema que altres investigadors ja havien detectat: la poca reproductibilitat de la congelació de l'aigua.

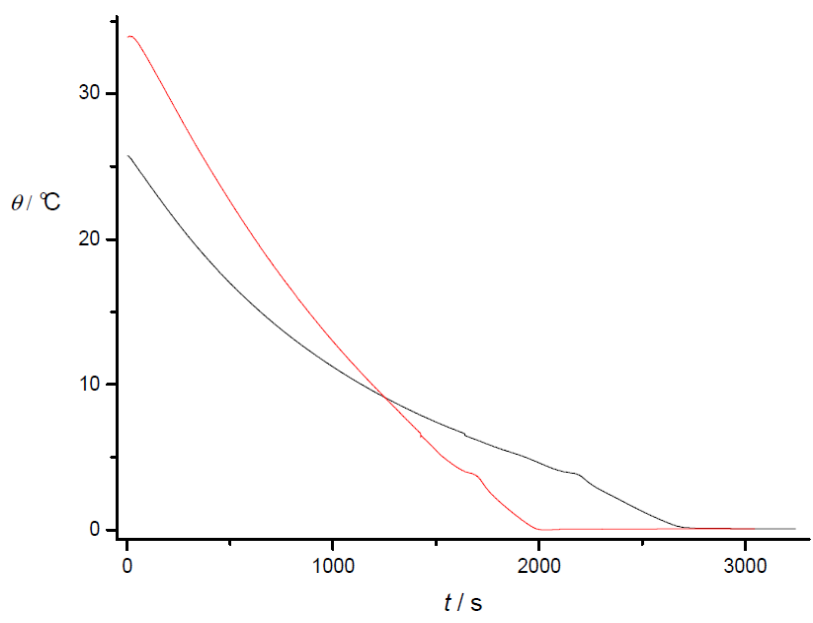

Figura 2. Refredament i congelació d'aigua desionitzada $(\mathrm{V}=30 \mathrm{ml}) \mathrm{a} \approx 25^{\circ} \mathrm{C}$ i $35^{\circ} \mathrm{C}$ en un vas de precipitats sense tapar i sense agitar. (Adaptat de l'article original.)

Finalment, l'autor conclou que la causa de l'efecte Mpemba és una combinació de dos factors: els corrents de convecció i el súper refredament. A continuació, es resumeixen breument els principals

https://store.sciencebuddies.org/Phys_p032/SBD-5210-

KIT/Mpemba-Effect-Science-Kit.aspx 
arguments que dóna Bregovic per justificar aquesta afirmació (tot i que, per a una visó més completa, es recomana llegir l'article original).

\section{Convecció induïda per gradients de temperatura}

Per tal d'entendre com s'origina la convecció, imaginem que s'introdueix una mostra d'aigua calenta en un frigorífic. La part de la mostra que es troba en contacte amb les parents del recipient es refreda més ràpidament que no pas la part interna, de manera que s'indueix un gradient de temperatura a l'interior de la mostra. Això fa que es generi un corrent de convecció, i com més gran sigui el gradient de temperatura, més accentuada serà la convecció, i més ràpidament tindrà lloc el refredament general de la mostra (assumint que la convecció es manté al llarg de tot el procés de refredament).

A banda d'aquest raonament, Bregovic fa una sèrie d'experiments que consisteixen a refredar mostres d'aigua que estan inicialment a temperatures diferents $\left(\mathrm{a} \approx 25^{\circ} \mathrm{C}\right.$ i $\left.35^{\circ} \mathrm{C}\right)$. A partir de les corbes de refredament, observa que aquestes tendeixen a una lleugera estabilització al voltant dels $4^{\circ} \mathrm{C}$, valor que correspon a la temperatura en què l'aigua freda es torna menys densa que l'aigua calenta. En aquest punt, argumenta, la convecció es veuria impedida pel fet que el gradient de densitat és zero, $\mathrm{i}$ a partir d'aquí, a mesura que l'aigua freda s'anés tornant menys densa, pujaria per la columna d'aigua induint corrents de convecció durant el procés de refredament, la qual cosa faria que la mostra se seguís refredant ràpidament just després dels $4^{\circ} \mathrm{C}$.

D'altra banda, també compara les corbes de refredament corresponents $a$ :

- Dues mostres de $30 \mathrm{ml}$ d'aigua que inicialment estan a $25^{\circ} \mathrm{C}$ i $35^{\circ} \mathrm{C}$ sense agitació (Figura 2).

- Dues mostres de $30 \mathrm{ml}$ d'aigua que inicialment estan a $25^{\circ} \mathrm{C}$ i $35^{\circ} \mathrm{C}$ amb agitació magnètica (Figura 3).

Si comparem les figures 2 i 3 , i ens fixem en quan arriben als $0^{\circ} \mathrm{C}$ (i s'estabilitzen) les mostres calenta i freda, s'observa que l'efecte Mpemba és significativament menor a la Figura 3 , és a dir, quan la mostra és agitada durant el procés de refredament. Aquest fet confirma la importància dels corrents convectius però no pot ser suficient, ja que fins i tot amb agitació es congela abans la mostra que inicialment està més calenta.

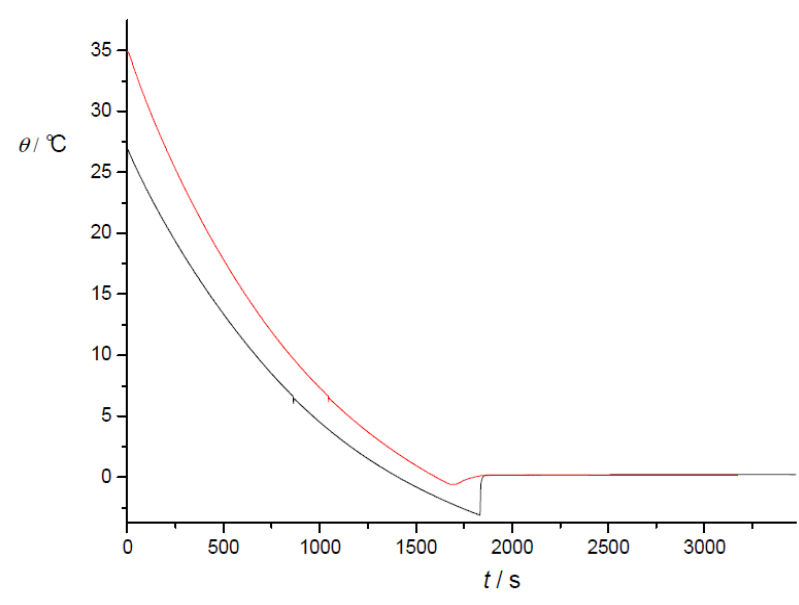

Figura 3. Refredament i congelació d'aigua desionitzada $(\mathrm{V}=30 \mathrm{ml}) \mathrm{a} \approx 25^{\circ} \mathrm{C}$ i $35^{\circ} \mathrm{C}$ en un vas de precipitats amb agitació . (Adaptat de l'article original.)

\section{Súper refredament}

El súper refredament és el procés de refredar un líquid per sota del seu punt de congelació sense que aquest es faci sòlid. En el cas de l'aigua, per exemple, seria possible súper refredar-la fins a gairebé $-42^{\circ} \mathrm{C}$, malgrat que el seu punt de congelació sigui de $0^{\circ} \mathrm{C}$.

El súper refredament, tot i ser necessari per tal que es pugui formar el primer cristall de gel, de vegades no es detecta perquè té lloc de forma localitzada a les parets del recipient. En aquest sentit, encara que la temperatura estigui molt per sota dels $0^{\circ} \mathrm{C}$ en el punt on comencen a formar-se els cristalls de gel (és a dir,a les parets del recipient), això pot no ser apreciable a la part central de la mostra [3, 4]. L'autor suggereix que petits canvis en la temperatura de súper refredament poden influir en el temps necessari per congelar una mostra. De fet, aquest fenomen seria, al seu parer, un dels principals causants de la poca reproductibilitat en les investigacions sobre congelació de l'aigua.

De tota manera, diu, el problema principal no està en explicar com el súper refredament afecta el temps de congelació, sinó com la temperatura inicial pot afectar el súper refredament. En aquest sentit, i segons un estudi sobre factors que determinen la temperatura de súper refredament, aquesta es pot veure modificada si la mostra s'escalfa prèviament, en funció de la seva naturalesa i del recipient [5]. Això permetria que dues mostres d'aigua extretes de la mateixa ampolla poguessin diferir significativament en les seves propietats de súper refredament, i que l'efecte Mpemga pogués tenir lloc o no sota les mateixes condicions. 
L'article de Bregovic conclou, doncs, que el súper refredament i la convecció són fenòmens que cal tenir en compte per explicar l'efecte Mpemba. Tot i així, la qüestió encara no està completament resolta, i caldrà explorar amb més detall aquest comportament de l'aigua. En paraules del mateix Bregovic, "un cop més, aquesta petita, simple molècula ens sorprèn i intriga amb la seva màgia".

\section{Conclusions}

Probablement el jove Erasto Mpemba no s'imaginava, quan anava a l'institut, que al cap de cinquanta anys encara no hi hauria una explicació clara a les seves observacions: que la barreja calenta per fer gelats es congelava abans que la barreja freda. El mateix Mpemba explica, en l'article conjunt amb Osborne, com va començar tot, que traduït al català vindria a ser així:

Al 1963, quan era al tercer curs a l'escola secundària de Magamba, Tanzània, solia fer gelats. Els nois a l'escola en fan bullint llet, barrejant-la amb sucre $i$ posant-ho tot a dins el congelador, després d'haver-ho deixat refredar gairebé a temperatura ambient. Molts nois en fan, i s'ha d'anar ràpid per tenir lloc al congelador.

Un dia, després de comprar llet a una senyora del poble, vaig començar a bullir-la. Un altre noi, que havia comprat una mica de llet per fer gelat, va córrer cap al congelador quan em va veure bullint la Ilet, $i$ va barrejar ràpidament la seva llet amb sucre $i$ la va abocar en una glaçonera sense bullir-la; així no perdria l'oportunitat. Jo sabia que, si m'esperava a que la llet bullida es refredés abans de ficar-la al congelador, perdria la darrera glaçonera que hi havia disponible, així que vaig decidir arriscar-me a espatllar el congelador introduint-hi la llet calenta. L'altre noi i jo vam tornar una hora i mitja més tard $i$ vam trobar que a la meva safata de llet hi havia gelat, mentre que a la seva només hi havia un líquid espès, que encara no s'havia congelat.

Li vaig preguntar al meu professor de física per què havia passat això, que la llet que estava calenta s'havia congelat primer, i la resposta que em va donar va ser "Et vas confondre, això no pot passar". Aleshores em vaig creure la seva resposta.

La insistència del jove Mpemba en reafirmar els seus resultats experimentals $i$ en voler saber per què tenia lloc aquest fenomen van provocar la burla dels seus companys i professors. Afortunadament, va haver-hi algú que es va prendre seriosament les seves observacions: el Dr. Osborne, que havia vin- gut de l'University College de Dar Es Salaam (Tanzània) a fer una xerrada a l'institut on estudiava en Mpemba. Què hauria passat si aquest professor també hagués donat per fet que el jove estava equivocat? Potser algú altre hauria publicat més endavant els resultats d'aquests experiments i ara no estaríem parlant de l'efecte Mpemba, sinó d'un efecte amb un nom molt menys exòtic. Sigui com sigui, la tenacitat del jove tanzà es va veure recompensada, i mitjançant el concurs, la Royal Society of Chemistry ha contribuït a difondre aquesta interessant història. Per cert, i tenint en compte com va anar tot plegat, trobo que va ser un detall molt maco que es donés el nom d'efecte Mpemba a aquest comportament de l'aigua; li haurien pogut dir efecte Osborne, i seria més fàcil de pronunciar.

\section{Bibliografia}

[1] Mpemba, E. B.; Osborne, D. G. (1969) "Cool?" Physics Education (Institute of Physics) 4 (3): 172-175.

doi: $10.1088 / 0031-9120 / 4 / 3 / 312$

Enllaç a una versió de l'article editada per l'autor:

http://www.rsc.org/images/Cool-Mpemba-

Osborne1969_tcm18-222099.pdf

[2] Bregovic, N. (2012), Mpemba effect from a viewpoint of an experimental physical chemist. http://www.rsc.org/images/nikola-bregovicentry_tcm18-225169.pdf

[3] Auerbach, D. (1995), "Supercooling and the Mpemba effect: When hot water freezes quicker than cold" Am. J. Phys, 63 (10): 882-885.

[4] Thomas, J.H. (2007), The Mpemba Effect: Studying the effects of initial temperature, evaporation, and dissolved gasses on the freezing of water

http://www3.wooster.edu/physics/jris/Files/Thom as_Web_article.pdf

[5] Dorsey, N.E. (1948), Trans. Am. Phil. Soc., 38, 247-326. 